\title{
Bladder Alveolar Soft Part Sarcoma
}

National Cancer Institute

\section{Source}

National Cancer Institute. Bladder Alveolar Soft Part Sarcoma. NCI Thesaurus. Code C156277.

An alveolar soft part sarcoma involving the bladder. 